\title{
SURFACE WAVES OF FINITE SIZE ELECTROMAG- NETIC BAND GAP WOODPILE STRUCTURES
}

\author{
I. Ederra, J. C. Iriarte, and R. Gonzalo
}

Electrical and Electronic Engineering Department

Universidad Pública de Navarra, Pamplona 31006, Spain

\section{P. de Maagt}

Electromagnetics Division

European Space Research and Technology Centre, ESTEC

P. O. Box 299, AG Noordwijk 2201, The Netherlands

\begin{abstract}
This paper studies the surface modes at the interface of finite size Electromagnetic Band Gap (EBG) woodpile structures. The impact of different types of woodpile terminations on the properties of these surface modes is analyzed. For all the studied terminations there exist surface modes which must be taken into account when designing components based on this EBG structure.
\end{abstract}

\section{INTRODUCTION}

Electromagnetic Band Gap (EBG) structures, also known as Photonic Crystals or Photonic Band Gap Materials [1] are periodic structures which do not allow the propagation of electromagnetic waves within certain frequency ranges. The possibility of engineering the properties of these materials has paved the road for its application in different fields and frequency ranges [2].

The woodpile or layer-by-layer structure is one of the most popular EBG structures [3,4]. Among the advantages that lead to its popularity are its large bandwidth, robustness and relative simple fabrication. As a matter of fact, prototypes have been realized from the microwave and millimeter ranges up to optical frequencies [5-10] for applications such as antennas [11-13], millimeter wave components [14-16] or optical communications [17-19].

Received 18 November 2010, Accepted 24 December 2010, Scheduled 15 January 2011

Corresponding author: Inigo Ederra (inigo.ederra@unavarra.es). 
One of the proposed uses of this EBG structure is in the field of antennas. The woodpile can be used as antenna substrate, placing a planar antenna on top of its surface $[11,12]$. Since no electromagnetic radiation can propagate within its bandgap the structure would behave as a reflector for the antenna radiation, therefore enhancing its radiation features. Furthermore, the mutual coupling between antennas is reduced by this type of configuration [20]. In addition to this the woodpile can be used to build waveguides which change its dimensions near the edge, creating horn-like antennas [13].

The use of the woodpile as antenna substrate is based on the idea that the non infinite woodpile structure will keep its bandgap unaffected by the fact that the structure has been truncated. However, when an EBG structure is truncated, the translation symmetry is ruined in the direction perpendicular to the termination plane. As a consequence, surface modes can be created in the interfaces between the EBG structure and the other medium, usually air [2131]. These surface modes propagate parallel to this interface and have electromagnetic field distributions which decay in both the air and the EBG directions. Since decaying inside the EBG structure is required, these substrate modes only exist in frequencies inside the gap of the bulk EBG structure as it is explained, e.g., in [31].

Due to its periodicity, the termination of the EBG structure is arbitrary, although for real structures it is usually determined by the manufacturing procedure. In a layer-by-layer fabrication approach, which is the standard procedure used to create the woodpile structure, the termination is given by the surface of a complete layer. In [21], it is postulated that for any EBG structure a termination which allows for the existence of surface modes always exists. In the woodpile case, these different terminations have been created by adding additional bars [28], which allowed to create high $Q$ cavities.

Moreover, for antenna substrate applications, a dielectric substrate, on which the antenna is printed, is also present on top of the woodpile. The thickness of this substrate will have an impact on the frequency range of these surface modes. Similar effects have been found in the performance of 1D finite Bragg stacks, which are highly dependent on the stack sequence close to its boundary [26].

In this paper, the electromagnetic behavior of the terminated woodpile structures will be presented. First, the main features of the woodpile structure will be shown. The effect of the surface termination for the silicon woodpile will be studied in Sections 3 and 4. Since a dielectric substrate placed on the surface is a standard configuration when using the woodpile as an antenna substrate, its effect is presented in Section 5. The effect of the woodpile thickness is analyzed in 
Section 6. Finally, the conclusions are drawn in Section 7.

\section{PROPERTIES OF THE INFINITE WOODPILE STRUCTURE}

The woodpile or layer-by-layer EBG structure was first proposed by Ho [3] and Sözuer [4]. It is created by stacking layers of bars rotated $90^{\circ}$ with respect to the bars in the neighboring layers and shifted half a period with respect to those with the same orientation. The bars are usually square or rectangular, although similar behavior can be found with cylindrical bars [32].

For an infinite woodpile structure the band gap width and position depends on the material of the bars and on their dimensions. Of particular relevance is the ratio between the stacking period and the in plane period, $c$, see Fig. 1. This parameter can be used to optimize the bandwidth of the structure. In particular, in the case of square silicon bars, the maximum relative band gap bandwidth, $21 \%$, is obtained for $c=1.22$ [5]. This value will be used in the rest of the paper. Within the band gap frequency range no electromagnetic radiation can propagate inside the structure, as shown in its band diagram, Fig. 1.

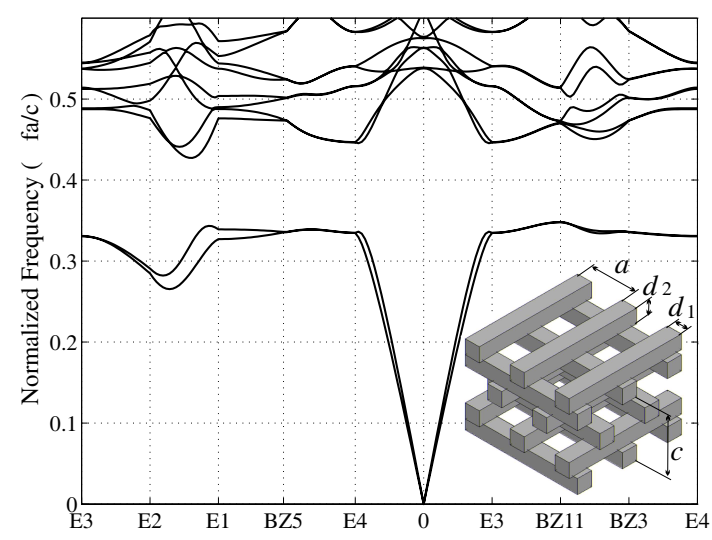

Figure 1. Band diagram of the optimized bandwidth silicon woodpile $\left(c=1.22 ; d_{1}=d_{2}=c a / 4\right)$. The horizontal axis represents $3-\mathrm{D}$ wavevectors, the value of which traces a path around the boundary of the irreducible Brillouin zone of the lattice. The inset shows the definition of the woodpile parameters.

\section{Reproduced courtesy of The Electromagnetics Academy}

Regarding the dielectric material used to create the bars, the bandwidth increases along with its dielectric constant [33]. Simultaneously, the dimensions of the structure for a certain central 
frequency of the gap decrease. Hence, the use of high dielectric constant materials has been proposed in order to create compact broadband woodpile structures [5]. However, in this paper, we will focus on silicon, since it is the most used material for this applications.

\section{FINITE THICKNESS SILICON WOODPILE}

The behavior of an infinite periodic woodpile has been introduced in the previous section. However, in any real application, the dimensions of the manufactured woodpile will be finite. The structure will have a boundary, which under certain circumstances can support surface modes.

If an EBG structure is finite in one dimension the band structure changes with respect to the infinite structure case. In the semi-infinite structure case, surface modes can exist at the interface between the EBG and the surrounding materials, usually air. These surface modes depend on the surface termination and on the direction of propagation that is considered [21]. In our study, a finite thickness woodpile structure will be considered, i.e., the number or periods in the stacking direction, $z$ axis according to Fig. 2, will be finite. The woodpile will remain infinitely periodic in the other directions.

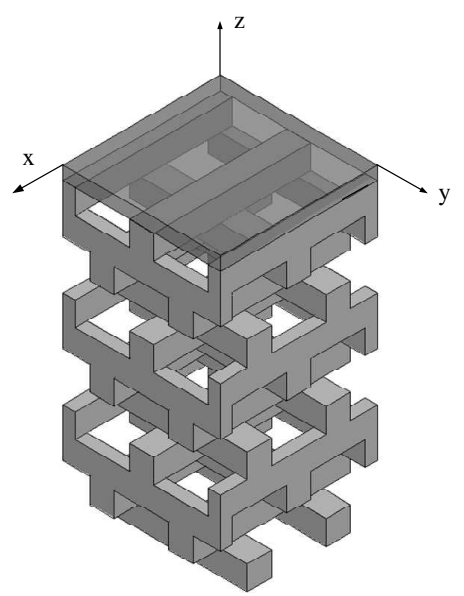

Figure 2. Finite thickness woodpile structure. The quartz substrate used in some of the studied cases is shown in dark grey. Reproduced courtesy of The Electromagnetics Academy

In order to analyze these finite thickness EBG structures the so-called supercell method [31] was used. Since the structure is not periodic anymore in the $z$ direction, an artificial periodicity is created 
by adding an air layer on top of the EBG structure. This layer must be thick enough in order to guarantee that the decaying fields in the interface are not affected by the artificial periodicity. Periodic boundary conditions are assumed in the $x$ and $y$ directions. The band diagrams of these structures were computed by preconditioned conjugate-gradient minimization of the block Rayleigh quotient in a planewave basis [34].

Although finite EBG structures can be finished at any location, the most natural termination of a woodpile structure consists on having a complete layer of bars as top layer. In these cases, the overall thickness of the woodpile can be an integer number of periods or some bar layers could be eliminated. In addition to this, different terminations can be created by cutting the bars of the top layer at different heights.

In order to compute the band diagram of the finite thickness woodpile and obtain the propagation characteristics of the surface modes, propagation directions parallel to the woodpile-air interface have been considered. In this case, the woodpile bars of the top layer determine the main propagation directions for the modes, i.e., parallel and perpendicular to them. According to Fig. 2, these correspond to the $x$ and $y$ directions respectively. If the woodpile thickness is an integer number of periods the two air-woodpile interfaces are formed by bars in perpendicular directions and therefore both directions are equivalent and the band diagrams will be identical. For the sake of clarity, we will focus on the behavior of the top surface in Fig. 2. In this surface, the $x$ direction corresponds to the direction of the bars.

As regards to the number of woodpiles periods, structures consisting of four of them have shown to present attenuation levels large enough for many applications [5]. Consequently this will be the initial configuration analyzed. The band diagrams obtained in this case for propagation in the $x$ and $y$ directions are shown in Fig. 3. In this figure, three types of modes are present: surface modes which propagate along the $x$ and $y$ directions, labelled as X1, X2 and Y1, and slab modes, created by the finite thickness of the woodpile. The analysis presented here does not include all the propagation directions. However, the considered propagation directions are the most representative ones and give enough information about the behavior of finite woodpiles.

The surface modes correspond to states which are decaying in both the air and the EBG structure and therefore must lay within the gap of the EBG structure and outside the radiation area. The slabs modes are located outside the bandgap of the infinite woodpile or at its boundary, so that they posses a standing wave pattern in the EBG slab, whereas they are decaying in the air. These slab modes propagate in both propagation directions. 


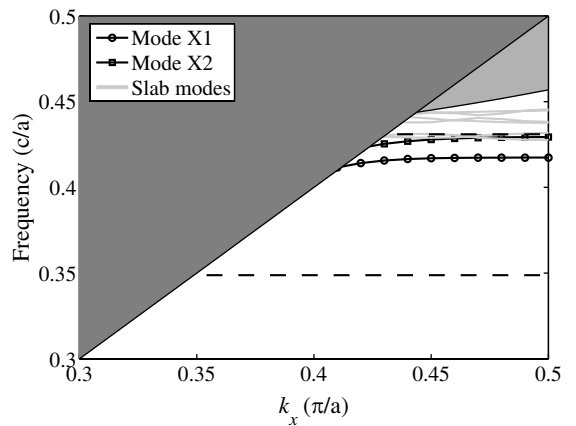

(a)

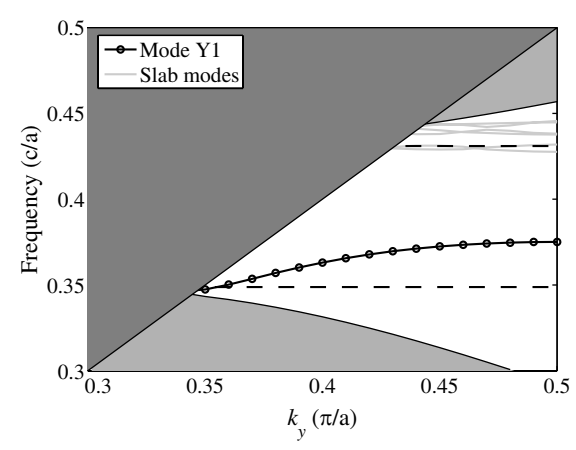

(b)

Figure 3. Dispersion diagram of a 4 period woodpile structure for propagation in the $x$ and $y$ directions. The dashed lines represent the limits of the infinite woodpile full band gap. The white area represents the band gap of the infinite woodpile structure in these propagation directions. The dark grey area is the radiation zone.

\section{Reproduced courtesy of The Electromagnetics Academy}

In this diagram, only those modes that lay inside the gap of the infinite woodpile structure in these propagation directions, represented as the white area, are shown. However, the full gap of the woodpile, indicated by the dashed lines, is smaller than the gap in this direction. As a consequence, some of these modes, in particular most of the slab modes, are outside of the full gap and will not affect the performance of the woodpile, provided it is used in its usual working frequency range, i.e., frequencies inside the full gap.

Focusing on the surface modes, the frequency range of mode X2 is very close to the upper limit of the full gap. Mode X1 is slightly lower in frequency, and mode Y1 covers the lower area of the gap. As a consequence, the central area of the gap is free of surface modes and the behavior of the woodpile would not be affected by its finite dimensions.

Besides these 3 surface modes, there are four slab modes, shown in grey in Fig. 3, which are outside of the full gap of the woodpile. Therefore they would not interfere with the behavior of the EBG. However, under some surface terminations that will be studied in the following sections some of these modes can shift to lower frequencies, becoming surface modes and entering the full gap area.

In order to get some insight into the properties of these modes their field distribution at the point $k=0.5 \pi / a$ in the $x$ and $y$ directions is represented in Fig. 4. These correspond to the following operating normalized frequencies: 0.3751 (mode Y1), 0.4174 (mode X1), 0.4294 (mode X2) and 0.4276 (mode S1). For the three surface modes the 

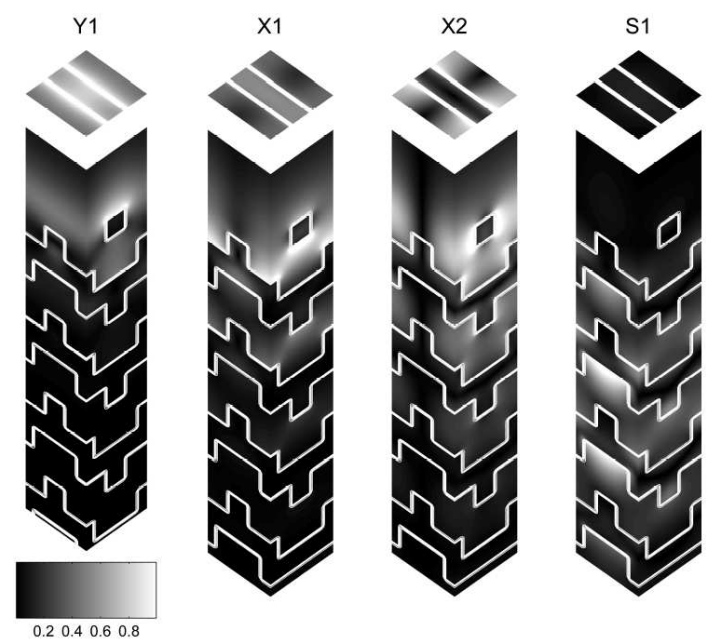

Figure 4. Electric field magnitude of the modes of a truncated silicon woodpile at the $k=0.5 \pi / a$ point.

Reproduced courtesy of The Electromagnetics Academy

$\mathrm{Y} 1$

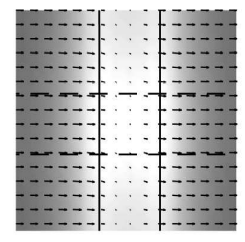

$\mathrm{X} 1$

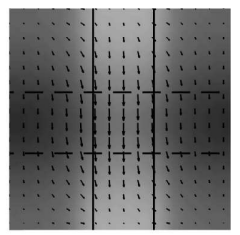

$\mathrm{X} 2$

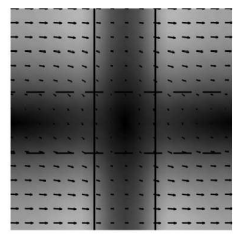

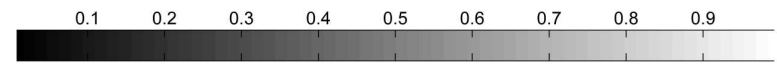

Figure 5. Electric field distribution at the air-quartz interface for the different surface modes of a truncated silicon woodpile at the $k=0.5 \pi / a$ point. The black lines represent the edges of the woodpile bars.

\section{Reproduced courtesy of The Electromagnetics Academy}

field is confined in the interface between the woodpile and the air. For the sake of comparison the first slab mode is represented as well, S1 diagram. In this case, the mode is located inside the woodpile slab.

Of particular importance is the mode field distribution and polarization at the air-woodpile interface. This polarization will determine the amount of excitation of these modes in case a source is placed close to this interface. In particular, as shown in Fig. 5, mode Y1 and X2 are mainly polarized along the $y$ direction, whereas mode $\mathrm{X} 1$ is polarized along the $x$ direction. 


\section{EFFECT OF THE SURFACE TERMINATION}

In the previous section, the analyzed woodpiles were terminated in a complete bar. However, any termination is possible and the last layer of bars can be different from the previous ones. In this case the dispersion diagrams obtained for different sizes of the woodpile bars of the last layer have been analyzed. In particular, the bar thickness has been reduced and augmented in steps of $0.25 d_{1}$, where $d_{1}$ is the original thickness of the bars, as shown in Fig. 1.

In the case of the surface modes, the observed effect is the same in all cases, see Fig. 6. Increasing the size of the top bars increases the amount of dielectric constant and the dispersion curves of these modes shift to lower frequencies. Conversely, if the bar thickness is reduced the dispersion curves tend to shift to higher frequencies. In this last case, for bars thinner than $0.75 d_{1}$ modes $\mathrm{X} 1$ and $\mathrm{X} 2$ are no longer supported as surface waves and become slab modes.

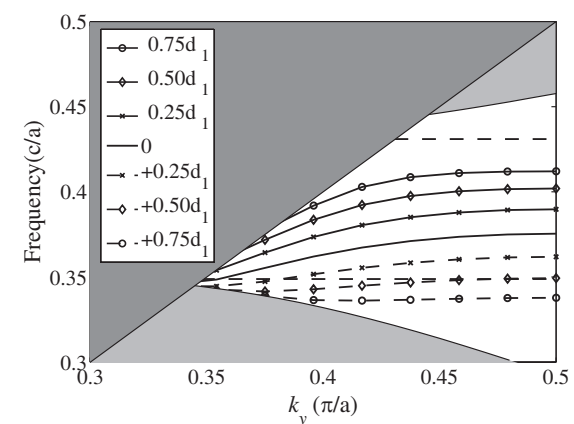

(a) Mode Y1

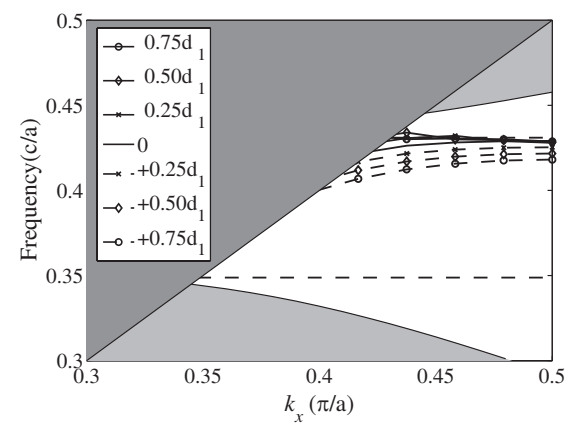

(c) Mode X2

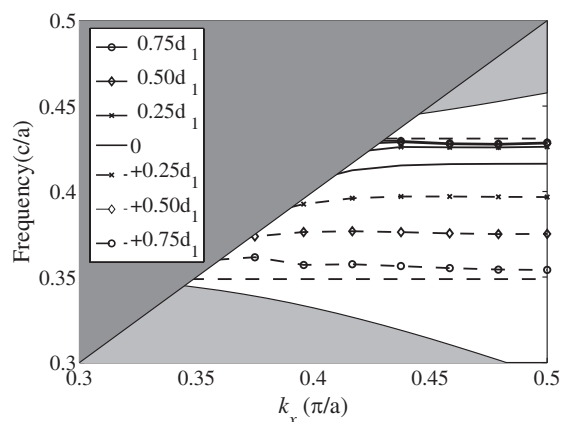

(b) Mode X1

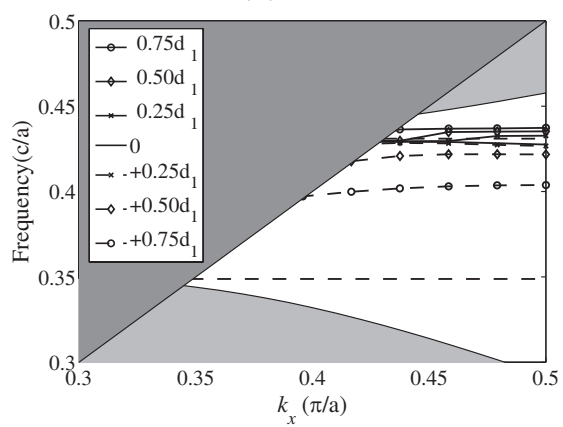

(d) Slab Mode 1

Figure 6. Dispersion diagram of the terminated woodpile structure as a function of the surface termination. The white area represents the band gap of the infinite woodpile structure in these propagation directions. The grey area is the radiation zone.

\section{Reproduced courtesy of The Electromagnetics Academy}


For the already existing slab modes the effect is the same: increasing the bar thickness makes them become surface modes, as shown in Fig. 6(d). This type of behavior is similar to that observed in other EBG structures [21].

\section{QUARTZ TERMINATED FINITE SILICON WOODPILE}

In antenna applications, when the woodpile structure is used as substrate for planar antennas, a thin dielectric substrate onto which the antennas are printed is required. The effect of this substrate has been studied for 2D EBG structures [35], leading to the conclusion that its effect was not relevant. However, only a transmission and reflection analysis was carried out, what might not be complete enough in order to fully characterize the effect of the substrate, since it is the near field of the antenna that interacts with the EBG structure.

In our case, the band diagram of the woodpile in combination with a quartz substrate has been studied. The presence of this dielectric substrate modifies the woodpile surface and consequently the band distribution changes. The dispersion diagram of the surface modes changes and some of the slab modes can turn into surface modes for thick enough quartz substrates.

The previously analyzed woodpile has been studied when a quartz layer is placed on its top surface. Quartz has been selected since it is the standard material used for this purpose in the (sub)millimeter wave range, where these structures find its main application. For the sake of realness, the thicknesses of this layer has the standard values for this type of application at these frequencies, i.e., 10-40 microns. Bearing in mind that the physical dimensions of a woodpile working around $500 \mathrm{GHz}$ (central frequency of the gap) are: $a=232.9$ microns, $d_{1}=d_{2}=71$ microns [5], the quartz substrate ranges between $0.043 a$ and $0.17 a$.

The effect of the quartz layer on the different modes is similar to that created when the bar thickness is increased, see Fig. 7. The first mode, which propagates in $y$ direction, mode $\mathrm{Y} 1$, shifts to lower frequencies when the substrate thickness increases, as shown in Fig. 7(a). This frequency shift is caused by the increase of the dielectric constant at the surface, where the field of the mode is confined. For substrates thicker than 20 microns the mode goes out of the full gap of the woodpile. Mode X1, Fig. 7(b), is affected by the quartz slab as well. In this case, its dispersion curves shift towards the centre of the gap keeping approximately the same slope. In particular, for 30 microns thick substrate the curve is at the centre of the full gap. The 
effect of the dielectric substrate on mode X2 is even larger, due to the higher concentration of electric field at the interface. Although the dispersion curve, shown in Fig. 7(c), is rather flat for thin substrates, it tends to steep for thicker substrates, covering the lower part of the gap. The quartz layer does not affect the slab modes, since they are located at the woodpile slab. As an example, the dispersion diagram of the first slab mode is shown in Fig. 7(d). The dispersion curve of this mode remains unaffected.

These results show that the use of a thick dielectric substrate modifies the band structure of the surface modes and must be taken into account when using this type of configurations, e.g., in antenna applications.

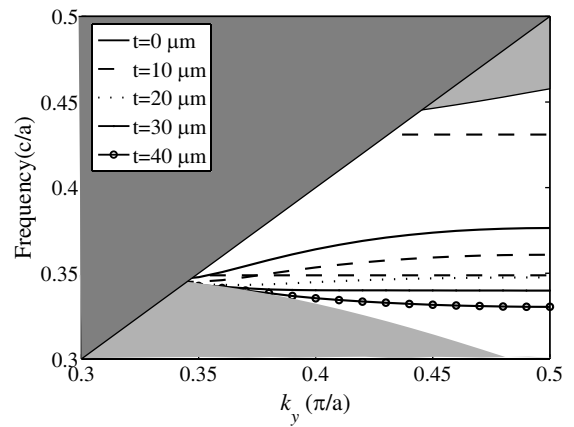

(a) Mode Y1

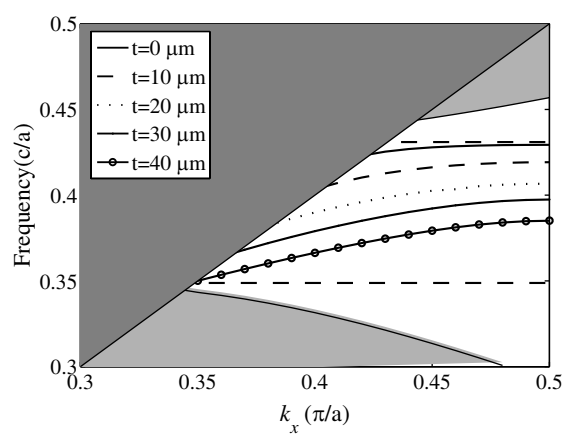

(c) Mode X2

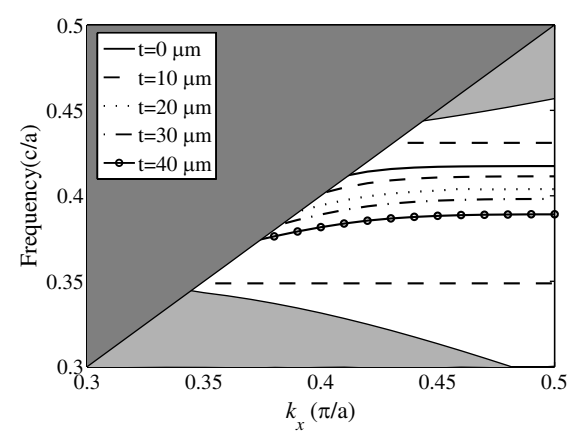

(b) Mode X1

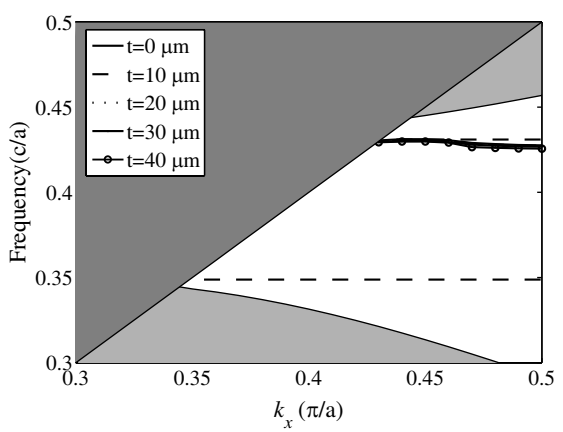

(d) Slab Mode 1

Figure 7. Dispersion diagram of the terminated woodpile structure as a function of the quartz substrate thickness. The white area represents the band gap of the infinite woodpile structure in these propagation directions. The grey area is the radiation zone.

\section{Reproduced courtesy of The Electromagnetics Academy}




\section{EFFECT OF THE NUMBER OF WOODPILE PERIODS IN THE STACKING DIRECTION}

The above presented results correspond to a 4 period $\mathrm{Si}$ woodpile. However, in practice, the number of periods can change and the woodpile performance as substrate will depend on its overall thickness. Actually, some of the modes previously discussed were slab modes, and its performance should depend on the woodpile thickness.

For this study, the woodpile will be covered by a 20 microns quartz slab. This value has been selected, since it is the average value of those previously used and it is a common value for (sub)millimeter wave applications. For this configuration, the number of woodpile periods in the stacking direction was varied from 2 to 6 and the effect of this

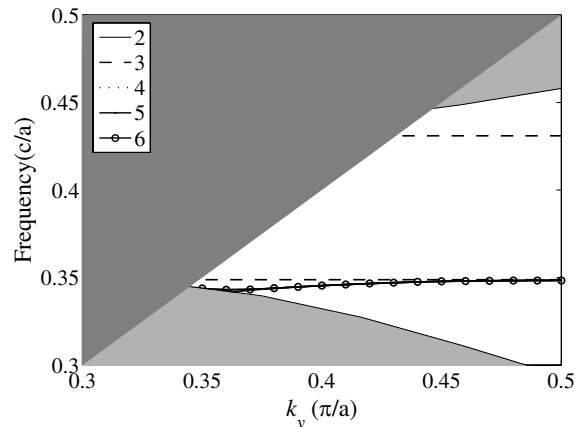

(a) Mode Y1

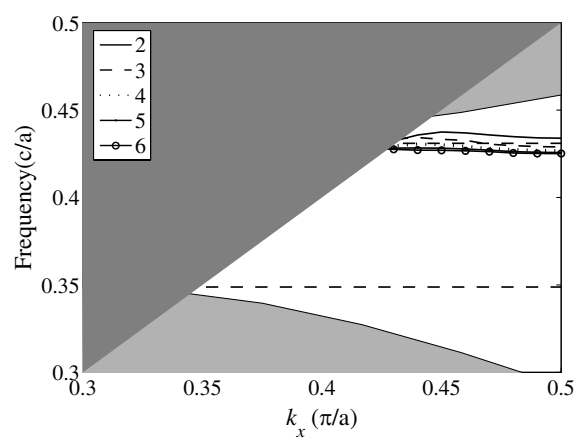

(c) Mode X2

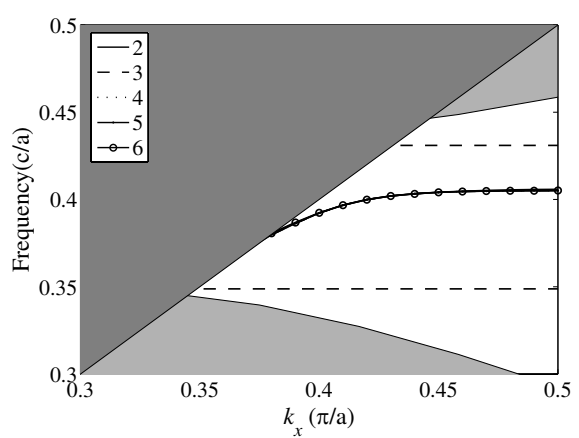

(b) Mode X1

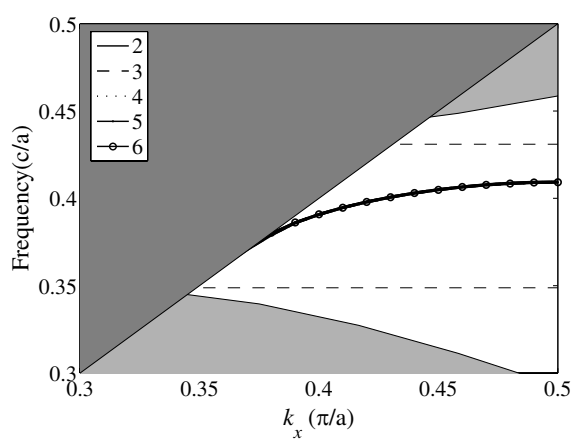

(d) Slab Mode 1

Figure 8. Dispersion diagram of a woodpile slab as a function of the number of periods in the stacking direction. The white area represents the band gap of the infinite woodpile structure in these propagation directions. The grey area is the radiation zone.

Reproduced courtesy of The Electromagnetics Academy 


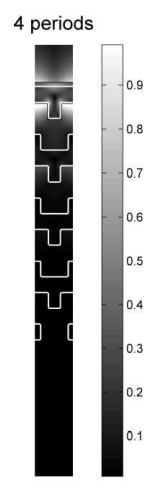

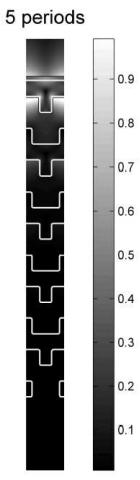

(a)
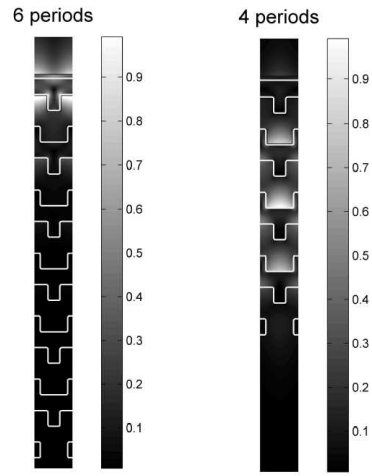
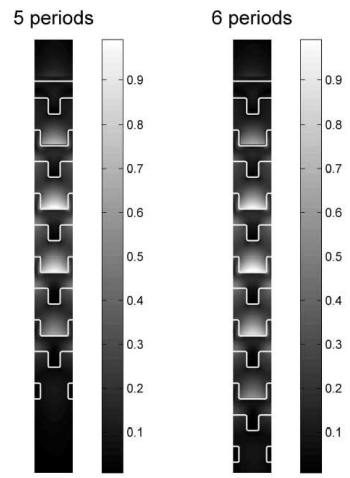

Figure 9. Field distribution at the $k=0.5 \pi / a$ point of (a) Mode X1 and (b) Slab Mode 1 as a function of the number of woodpile periods in the stacking direction. The operating normalized frequencies are 0.4051 (6 periods), 0.4051 ( 5 periods) and 0.4051 (4 periods) for mode $\mathrm{X} 1$ and 0.4251 ( 6 periods), 0.4257 ( 5 periods) and 0.4268 (4 periods) for mode S1.

\section{Reproduced courtesy of The Electromagnetics Academy}

variation is shown in Fig. 8. The dimensions of the woodpile are those mentioned in the previous Section.

Modes $\mathrm{Y} 1, \mathrm{X} 1$ and $\mathrm{X} 2$ are surface modes and its dispersion diagram does not change when the woodpile thickness is changed, as it is shown in Figs. 8(a) to (c). The modes decay inside the woodpile and therefore are not affected by the change of woodpile thickness. This effect is observed in Fig. 9 where the field distribution of mode $\mathrm{X} 1$ for 4,5 and 6 period thick woodpiles is presented. In all cases, the field distribution is the same. In the case, of the slab modes the dispersion curve changes, its properties depend on the thickness of the woodpile slab. As an example, the field distribution of Slab Mode 1 is shown in Fig. 9(b) where it can be observed that the field remains concentrated in the woodpile irrespective of the number of periods. Moreover, it tends to fill the whole slab, what explains the shift to lower frequencies of its dispersion curves.

\section{CONCLUSION}

The behavior of truncated woodpile structures has been studied. The finite dimensions of the woodpile allow the propagation of both surface waves, localized at the air-woodpile interface, and slab modes, due to its finite thickness. The existence of these surface modes is not a 
problem in itself. However, they must be taken into account when dealing with finite size structures in order to avoid being coupled.

In the case of a silicon woodpile three surface modes exist within the gap. Their dispersion curve depends on the surface termination and in some cases can be even at the centre of the bandgap. In addition to this, if a quartz substrate is placed on top of the woodpile the dispersion curve of these modes shift towards lower frequencies and can move them to the centre frequencies of the gap, interfering with the usual working frequency range of the EBG structure.

\section{ACKNOWLEDGMENT}

This work was supported by the Spanish Ministry of Science and Innovation Project Nos. TEC2009-11995 and CSD2008-00066.

\section{REFERENCES}

1. Joannopoulos, J. D., R. D. Meade, and J. N. Finn, Photonic Crystals: Molding the Flow of Light, Princeton University Press, 1995.

2. Lourtioz, J. M., V. Berger, J. M. Gerard, and H. Benisty, Photonic Crystals: Towards Nanoscale Photonic Devices, Springer, 2005.

3. Ho, K. M., C. T. Chan, C. Soukoulis, R. Biswas, and M. Sigalas, "Photonic band gaps in three dimensions: New layer-by-layer periodic structure," Solid State Comm., Vol. 89, 413-416, 1994.

4. Sözuer, H. S. and J. P. Dowling, "Photonic band calculations for woodpile structure," J. Mod. Opt., Vol. 43, 231-234, 1994.

5. Martínez, B., I. Ederra, R. Gonzalo, B. Alderman, L. Azcona, P. G. Huggard, B. de Hon, A. Hussain, S. R. Andrews, L. Marchand, and P. de Maagt, "Manufacturing tolerance analysis, fabrication and characterisation of 3D submillimetre wave electromagnetic bandgap crystals," IEEE Trans. Microw. Theory Tech., Vol. 55, No. 4, 672-681, 2007.

6. Özbay, E., "Layer-by-layer photonic crystals from microwave to far-infrared frequencies," J. Opt. Soc. Amer. B, Vol. 13, No. 9, 1945-1955, 1996.

7. Gonzalo, R., B. Martínez, C. M. Mann, H. Pellemans, P. H. Bolivar, and P. de Maagt, "A low cost fabrication technique for symmetrical and asymmetrical layer by layer photonic crystal at submillimeter wave frequencies," IEEE Trans. Microw. Theory Tech., Vol. 50, No. 10, 2384-2393, 2002. 
8. Noda, S., K. Tomoda, N. Yamamoto, and A. Chutinan, "Full Three-dimensional photonic bandgap crystal at near-infrared wavelengths," Science, Vol. 289, No. 5479, 604-606, 2000.

9. Lin, S. Y., J. G. Fleming, D. L. Hetherington, B. K. Smith, R. Biswas, K. M. Ho, M. M. Sigalas, W. Zubrzycki, S. R. Kurtz, and J. Bur, "A three-dimensional photonic crystal operating at infrared wavelengths," Nature, Vol. 394, No. 6690, 251-252, 1998.

10. Lin, S. Y. and J. G. Fleming, "A three dimensional optical photonic crystal," J. Lightwave Technol., Vol. 17, No. 11, 19441947, 1999.

11. Gonzalo, R., I. Ederra, C. M. Mann, and P. de Maagt, "Radiation properties of terahertz dipole antenna mounted on photonic crystal," Electron. Lett., Vol. 37, No. 10, 613-614, 2001.

12. Ederra, I., R. Gonzalo, B. E. J. Alderman, P. G. Huggard, B. P. de Hon, M. C. van Beurden, A. Murk, L. Marchand, and P. Maagt, "Electromagnetic bandgap based planar imaging array for $500 \mathrm{GHz}, "$ IEEE Trans. Microw. Theory Tech., Vol. 56, No. 11, 2256-2265, 2008.

13. Weily, A. R., K. P. Esselle, and B. C. Sanders, "Photonic crystal horn and array antennas," Phys. Rev. E, Vol. 68, No. 1, 0166091-016609-6, 2003.

14. Ederra, I., L. Azcona, B. E. J. Alderman, A. Laisnè, R. Gonzalo, C. M. Mann, D. N. Matheson, and P. de Maagt, "A $250 \mathrm{GHz}$ sub-harmonic mixer design using EBG technology," IEEE Trans. Antennas Propagat., Vol. 55, No. 11, 2974-2982, 2007.

15. Bayindir, M., E. Özbay, B. Temelkuran, M. M. Sigalas, C. M. Soukoulis, R. Biswas, and K. M. Ho, "Guiding, bending, and splitting of electromagnetic waves in highly confined photonic crystal waveguides," Phys. Rev. B, Condens. Matter, Vol. 63, No. 8, 081107, 2001.

16. Weily, A. R., T. S. Bird, K. P. Esselle, and B. C. Sanders, "Woodpile EBG phase shifter," Elec. Lett., Vol. 42, No. 25, 14631464, 2006.

17. Kawashima, S., K. Ishizaki, and S. Noda, "Light propagation in three-dimensional photonic crystals," Opt. Express, Vol. 18, No. 1, 386-392, 2010.

18. Sell, C., C. Christensen, J. Muehlmeier, G. Tuttle, Z. Y. Li, and K. M. Ho, "Waveguide networks in three-dimensional layer-bylayer photonic crystals," Appl. Phys. Lett., Vol. 84, No. 23, 46054607, 2004.

19. Kohli, P., C. Christensen, J. Muehlmeier, R. Biswas, G. Tuttle, 
and K.-M. Ho, "Add-drop filters in three-dimensional layer-bylayer photonic crystals using waveguides and resonant cavities," Appl. Phys. Lett., Vol. 89, No. 23, 231103, 2006.

20. Ederra, I., B. Martínez, A. B. Labajos, J. Teniente, R. Gonzalo, and P. de Maagt, "Experimental verification of the reduction of coupling between dipole antennas by using a woodpile substrate," IEEE Trans. Antennas Propagat., Vol. 54, No. 7, 2105-2111, 2006.

21. Meade, R. D., K. D. Brommer, A. M. Rappe, and J. D. Joannopoulos, "Electromagnetic Bloch waves at the surface of a photonic crystal," Phys. Rev. B, Vol. 44, No. 19, 10961-10964, 1991.

22. Feng, S., H.-Y. Sang, Z.-Y. Li, B.-Y. Cheng, and D.-Z. Zhang, "Sensitivity of surface states to the stack sequence of onedimensional photonic crystals," J. Opt. A: Pure Appl. Opt., Vol. 7, No. 8, 374-381, 2005.

23. Zhang, X., L. M. Li, Z. Q. Zhang, and C. T. Chan, "Surface states in two-dimensional metallodielectric photonic crystals studied by a multiple-scattering method," Phys. Rev. B, Vol. 63, No. 12, 125114, 2001.

24. Vlasov, Y. A., N. Moll, and S. J. McNab, "Observation of surface states in a truncated photonic crystal slab," Opt. Lett., Vol. 29, No. 18, 2175-2177, 2004.

25. Robertson, W. M. and M. S. May, "Surface electromagnetic wave excitation on one-dimensional photonic band-gap arrays," Appl. Phys. Lett., Vol. 74, No. 13, 1800-1802, 1999.

26. Ramos-Mendieta, F. and P. Halevi, "Surface electromagnetic waves in two-dimensional photonic crystals: Effect of the position of the surface plane," Phys. Rev. B, Vol. 59, No. 23, 15112-15120, 1999.

27. Qiu, M. and S. He, "Surface modes in two-dimensional dielectric and metallic photonic band gap structures: A FDTD study," Phys. Lett. A, Vol. 282, 85-91, 2001.

28. Ishizaki, K. and S. Noda, "Manipulation of photons at the surface of three-dimensional photonic crystals," Nature, Vol. 460, 367$371,2009$.

29. Eyni, Z., S. Roshan Entezar, A. Namdar, and H. Tajalli, "Tamm states of a nonlinear slab sandwiched between a uniform medium and a one-dimensional photonic crystal," Progress In Electromagnetics Research Letters, Vol. 18, 115-124, 2010.

30. Gaspar-Armentaa, J. A., F. Villa, and T. Lpez-Ros, "Surface waves in finite one-dimensional photonic crystals: mode coupling," 
Opt. Commun., Vol. 216, 379-384, 2003.

31. Ramos-Mendieta, F. and P. Halevi, "Electromagnetic surface modes of a dielectric superlattice: The supercell method," J. Opt. Soc. Am. B, Vol. 14, No. 2, 370-381, 1997.

32. Özbay, E., A. Abeyta, G. Tuttle, M. Tringides, R. Biswas, C. T. Chan, C. M. Soukoulis, and K. M. Ho, "Measurement of a three-dimensional photonic band gap in a crystal structure made of dielectric rods," Phys. Rev. B, Vol. 50, No. 3, 1945-1948, 1994.

33. Gonzalo, R., B. Martínez, and P. de Maagt, "The effect of dielectric permittivity on the properties of photonic bandgap devices," Microw. Opt. Technol. Lett., Vol. 23, No. 2, 92-95, 1999.

34. Johnson, S. G. and J. D. Joannopoulos, "Block-iterative frequency-domain methods for Maxwell's equations in a planewave basis," Opt. Express, Vol. 8, No. 3, 173-190, 2001.

35. Reynolds, A. L., H. M. H. Chong, I. G. Thayne, J. M. Arnold, and P. de Maagt, "Analysis of membrane support structures for integrated antenna usage on two-dimensional photonic-bandgap structures," IEEE Trans. Microw. Theory and Tech., Vol. 49, No. 7, 1254-1261, 2001. 